\title{
First-Principles Study of Electronic Structure and Optical Properties of Silicon/Carbon Nanotube
}

\author{
Wenyu Wang ${ }^{1}$, Jiangang Xu${ }^{1}$, Yunguang Zhang1* ${ }^{*}$ Guixia Li $^{2}$ \\ ${ }^{1}$ School of Science, Xi'an University of Posts and Telecommunications, Xi'an, China \\ ${ }^{2}$ School of Science, China University of Petroleum, Qingdao, China \\ Email: ${ }^{\star}$ zygsr2010@163.com
}

How to cite this paper: Wang, W.Y., Xu, J.G., Zhang, Y.G. and Li, G.X (2017) First-Principles Study of Electronic Structure and Optical Properties of Silicon/Carbon Nanotube. Computational Chemistry, 5, 159-171.

https://doi.org/10.4236/cc.2017.54013

Received: September 21, 2017

Accepted: October 24, 2017

Published: October 27, 2017

Copyright $\odot 2017$ by authors and Scientific Research Publishing Inc. This work is licensed under the Creative Commons Attribution International License (CC BY 4.0).

http://creativecommons.org/licenses/by/4.0/

\section{Open Access}

\begin{abstract}
A supercell of a nanotube formed by a carbon nanotube (CNT) and a silicon nanotube (SiNT) is established. The electronic structure and optical properties are implemented through the first-principles method based on the density functional theory (DFT) with the generalized gradient approximation (GGA). The calculated results show that $(6,6)-(6,6)$ silicon/carbon nanotubes (Si/CNTs) presented a direct band gap of $0.093 \mathrm{eV},(4,4)-(6,6)$ silicon/carbon nanotubes presented a direct band gap of $0.563 \mathrm{eV}$. The top of valence band was fundamentally determined by the $\mathrm{Si}-3 \mathrm{p}$ states and $\mathrm{C}-2 \mathrm{p}$ states, and the bottom of conduction band was primarily occupied by the C-2p states and $\mathrm{Si}-3 \mathrm{p}$ states in the $\mathrm{Si} / \mathrm{CNT}$. It was found that $(6,6)-(6,6) \mathrm{Si} / \mathrm{CNT}$ have smaller energy band gap and better conductivity. Besides, Si/CNTs have satisfactory absorption characteristics and luminous efficiency in ultraviolet band.
\end{abstract}

\section{Keywords}

Nanotubes, Electronic Structure, Optical Properties, First-Principles

\section{Introduction}

The discovery of CNTs since 1991 [1] have led to extensive research interest in one dimensional nanometer materials due to their unique properties and potential of applications [2] [3] [4] [5]. Recently, there has been a rapid growth research interests in advanced nanotechnology which gives great promise for achieving new sensing materials including high performance nanomaterials [6] and nanoscale eletronics devices [7]. Silicon, as the same group element with carbon in the periodic table of elements has similar structure with carbon, and 
has become one of hotspots in the field of nanometer materials research in recent years. Compared to other nanomaterials, SiNTs have more unique conduction properties. In addition, SiNTs have smaller size and excellent electrical properties, which enjoy broad application prospects in microelectronics and medicine and other fields. Some experimental researchers have attempted to develop the synthesis of SiNTs by a number of method such as chemical vapor deposition (CVD) method [8], template combined CVD [9]. In terms of theory, Luo et al. [10] studied the structures and electronic properties of pure $(5,5)$ and $(5,0)$ SiNTs with the first principles of density functional method, and found that the conductivity of SiNTs was connected with the chirality of its structure. According to the existing research findings [11], which shows that $(\mathrm{n}, 0)$ zigzag and $(\mathrm{n}, \mathrm{n})$ armchair SiNTs having $\mathrm{n} \geq 6$ are stable but those with $\mathrm{n}<6$ can be stabilized by internal or external adsorption of transition metal elements. Therefore, there are a lot of researches on SiNTs [12]. For example, Yang et al. [13] observed the electronic structure and the changes of optical properties by applying different degrees of pre-tension deformation on single-wall armchair $(6,6)$ SiNTs through density functional theory. Referring to other researches, as for single-walled armchair SiNTs, doping P can make the energy band gap narrow, enhance electrical conductivity [14], and doping $\mathrm{Al}$ can increase the optical absorption bandwidth of SiNTs, and improve the photoelectric properties [15]. Additionally, the SiNTs of different chirality have different levels of thermal stability, electrical conductivity and energy performance [16] [17]. With the further research work, more special nanotubes have been studied [18] [19]. A coaxial cylinderical heterojunction of carbon tubes, which consists of alternant bands of 5- and 7-membered rings, can be formed by one armchair (n, n) CNT and one zigzag $(2 \mathrm{n}, 0)$ CNT. The torsional mechanical properties are studied by using molecular dynamics method [20]. People have made some researches on the structure and properties of SiNTs, but few involve Si/CNTs. In the present paper, the electronic structure and optical properties of $(4,4)-(6,6) \mathrm{Si} / \mathrm{CNT}$ and $(4,4)-(6,6) \mathrm{Si} / \mathrm{CNT}$ s are studied.

In the present work, the electronic properties of Si/CNTs formed by a CNT and a SiNT are investigated with the method of the first-principles density functional theory. In the following, we first investigated electronic properties of $\mathrm{Si} / \mathrm{CNT}$ s in terms of energy band and density of states (DOS). And then the simulation results of optical properties of Si/CNTs are studied in Section 3. Finally, we conclude in Section 4. This work provided a theoretical basis for the application of SiNTs in photoelectric device.

\section{Simulation Model and Method}

\subsection{Model}

To study the structural feature and the electronic property of $(6,6)-(6,6)$ $\mathrm{Si} / \mathrm{CNTs}$ and $(4,4)-(6,6) \mathrm{Si} / \mathrm{CNTs}$, supercell are established. Periodic armchair nanotubes which both ends were not closed and infinitely long were adopted. 
Calculation was conducted on $a \times a \times c$ orthogonal supercell, and nanotubes infinitely extended along the repeating units of heterostructure at the direction $c$. In $\mathrm{Si} / \mathrm{CNT}$ s structure, SiNTs and CNTs occupied half in the heterostructure along the axial direction. Parameter and constant of supercell are set as $1 \times 1 \times 4$, $\alpha=\beta=90^{\circ}, \gamma=120^{\circ}$, respectively. $(6,6)-(6,6) \mathrm{Si} / \mathrm{CNT}$ include $48 \mathrm{Si}$ atoms and $48 \mathrm{C}$ atoms. $(4,4)-(6,6) \mathrm{Si} / \mathrm{CNT}$ include $32 \mathrm{Si}$ atoms and $48 \mathrm{C}$ atoms (ref. Figure 1).

\subsection{Method}

In this work, the first principles calculations for the computational method were carried out using CASTEP package to perform geometry optimization and the specific properties such as band energy and density of states (DOS). All the relaxation and electronic calculations were performed based on the DFT, within the Perdew-Burke-Ernzerhof (PBE) formulation of the generalized gradient approximation [21]. The convergence criteria for total energy and the cutoff energy of the plane-wave basis are set as $1.0 \times 10^{-6} \mathrm{eV}$ and $400 \mathrm{eV}$. Integration on the Brillouin zone is performed using special $k$ points generated by the Monkhorst-Pack [22] grid with the value of $1 \times 1 \times 1,1 \times 1 \times 1,2 \times 1 \times 1$ for SiNTs, 6 , $6)-(6,6) \mathrm{Si} / \mathrm{CNTs}$ and $(4,4)-(6,6) \mathrm{Si} / \mathrm{CNTs}$, respectively. Energy calculations were carried out in the reciprocal space. The valence electron states of all atoms considered: $\mathrm{C} 2 \mathrm{~s}^{2} 2 \mathrm{p}^{2}, \mathrm{Si} 3 \mathrm{~s}^{2} 3 \mathrm{p}^{2}$.

\section{Results and Discussion}

\subsection{Geometry Optimized}

In order to make the model energy tend to be stable, and more close to the real material, and ensure that parameters used in the simulation get accurate results, the initial model was first optimized. The geometry of the $(6,6) \operatorname{SiNTs},(6,6)-(6,6)$ $\mathrm{Si} / \mathrm{CNT}$ s and $(4,4)-(6,6) \mathrm{Si} / \mathrm{CNTs}$ are optimized by using a Broyden-Fletcher-Goldfarb-Shanno (BFGS) minimizer [23]. Optimization accuracy is set as Fine. The optimized lattice parameters and structure of SiNTs and Si/CNTs are shown in Table 1 and Figure 2.

Table 1. Optimized lattice parameters of $(6,6) \operatorname{SiNT},(6,6)-(6,6) \mathrm{Si} / \mathrm{CNT}$ and $(4,4)-(6,6)$ $\mathrm{Si} / \mathrm{CNT}$.

\begin{tabular}{|c|c|c|c|c|c|c|c|}
\hline Type & optimized & $\mathrm{a} / \AA$ & $\mathrm{b} / \AA$ & $\mathrm{c} / \AA$ & $\mathrm{d}_{\mathrm{Si}-\mathrm{Si}} / \AA$ & $\mathrm{d}_{\mathrm{C}-\mathrm{C}} / \AA$ & $\mathrm{d}_{\mathrm{C}-\mathrm{Si}} / \AA$ \\
\hline \multirow{2}{*}{$(6,6) \mathrm{SiNT}$} & before & 16.7542 & 16.7542 & 16.2120 & 2.34 & - & - \\
\hline & after & 15.7315 & 15.2161 & 15.6251 & 2.261 & - & - \\
\hline \multirow{2}{*}{$(6,6)-(6,6) \mathrm{Si} / \mathrm{CNT}$} & before & 16.4104 & 16.4104 & 15.7963 & 2.34 & 1.42 & - \\
\hline & after & 12.3433 & 12.3375 & 13.7579 & 2.362 & 1.431 & 1.895 \\
\hline \multirow{2}{*}{$(4,4)-(6,6) \mathrm{Si} / \mathrm{CNT}$} & before & 12.0560 & 12.0560 & 15.7963 & 2.34 & 1.42 & - \\
\hline & after & 11.3745 & 12.3977 & 12.4007 & 2.370 & 1.421 & 1.882 \\
\hline
\end{tabular}




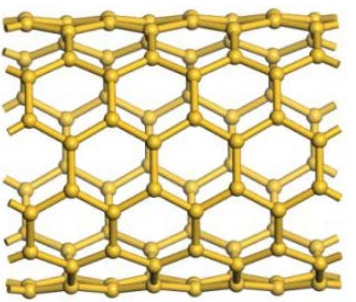

(a)

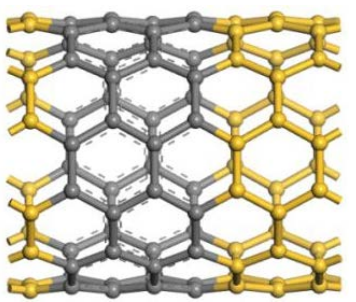

(b)

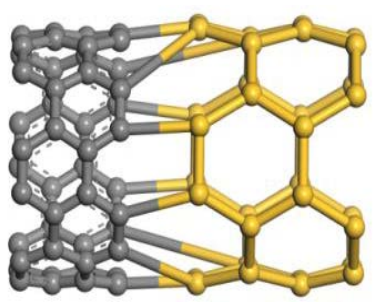

(c)

Figure 1. Structure schematics of $(6,6) \operatorname{SiNT},(6,6)-(6,6) \mathrm{Si} / \mathrm{CNT}$ and $(4,4)-(6,6)$ Si/CNT (The yellow ball: silicon atom; The grey ball: carbon atom). (a) $(6,6) \mathrm{SiNT}$; (b) $(6$, 6) - $(6,6) \mathrm{Si} / \mathrm{CNT}$; (c) $(4,4)-(6,6) \mathrm{Si} / \mathrm{CNT}$.

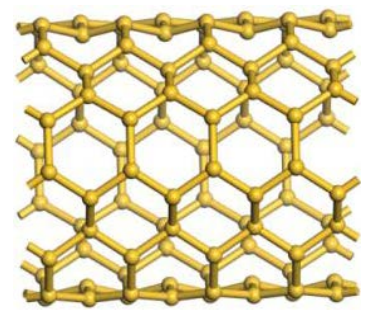

(a)

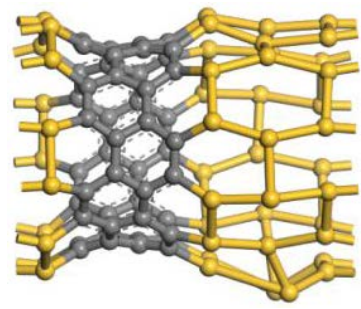

(b)

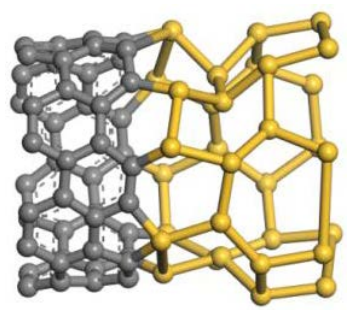

(c)

Figure 2. Optimized structure schematics of $(6,6) \mathrm{SiNT},(6,6)-(6,6) \mathrm{Si} / \mathrm{CNT}$, and $(4,4)$ - $(6,6) \mathrm{Si} / \mathrm{CNT}$. (a) $(6,6) \mathrm{SiNT}$; (b) $(6,6)$ - $(6,6) \mathrm{Si} / \mathrm{CNT}$; (c) $(4,4)-(6,6) \mathrm{Si} / \mathrm{CNT}$.

In the process of optimization, the change of internal bond length and bond angle of nanotubes changed the cell volume, but the optimized Si/CNTs crystal cell parameters were close. Si-Si bond in SiNTs has bigger length fluctuation and lower chemical displacement degree. Compared with the smooth structure of CNTs surface, the surface of SiNTs appeared folding structure, and Si/CNTs surface presented some deformation. By comparing with the model in Figure 1, it showed that optimization had a greater influence on SiNTs, while slightly affected CNTs part.

\subsection{Electronic Structures}

To have a understanding of the electronic properties of Si/CNTs, the band structure and DOS for the single-walled armchair $(6,6)$ SiNTs, $(4,4)-(6,6)$ $\mathrm{Si} / \mathrm{CNTs}$ and $(6,6)-(6,6) \mathrm{Si} / \mathrm{CNT}$ s were calculated and presented in Figures 3-5. Figure 3(a) shows the band structure of the single-walled armchair $(6,6)$ SiNTs. The bottom of the conduction band of the nanotube locates at the $G$ point of its Brillouin zone, and the top of valence band lies between the point $\mathrm{G}$ and F. Besides, the coordinate of them are $(0,0)$ and $(0.14,0)$, respectively, indicating that the nanotubes is an indirect gap semiconductor. The obtained band gap is $0.314 \mathrm{eV}$, which is highly in consistent with the result of Yang's [24].

The valence band of $(6,6)$ SiNTs mainly comprises the lower valence band of $-12 \mathrm{eV}-5 \mathrm{eV}$ and upper valence band of $-5 \mathrm{eV}-0 \mathrm{eV}$. The top of valence band was fundamentally determined by the Si-3p states and the bottom of conduction band was primarily occupied by the $\mathrm{Si}-3 \mathrm{~s}$ states and $\mathrm{Si}-3 \mathrm{p}$ states, showing that 


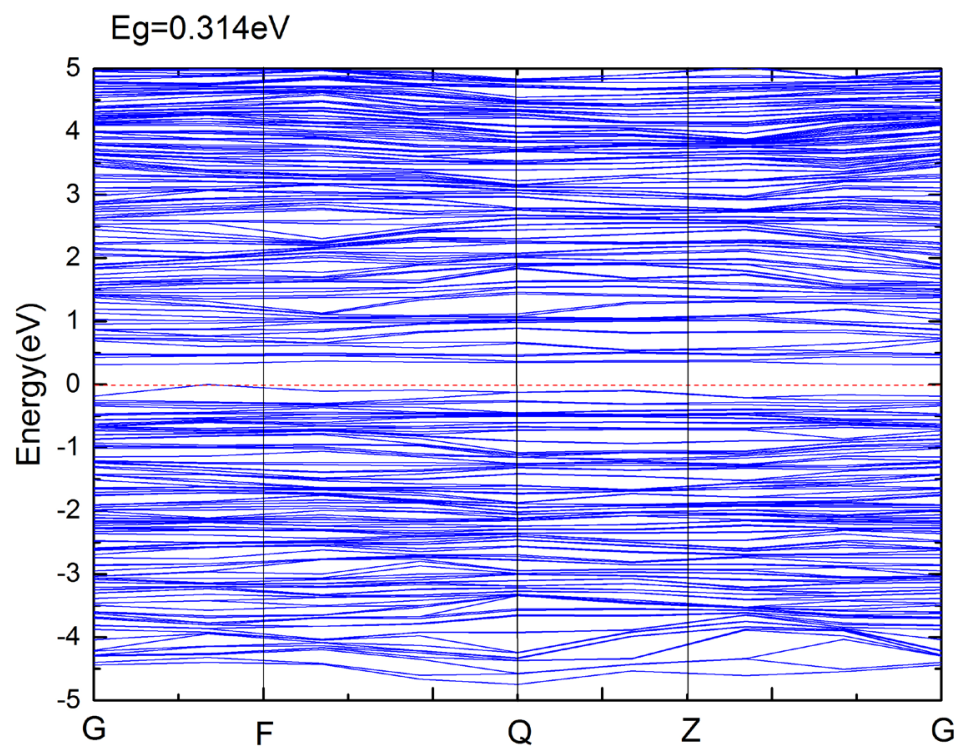

(a)

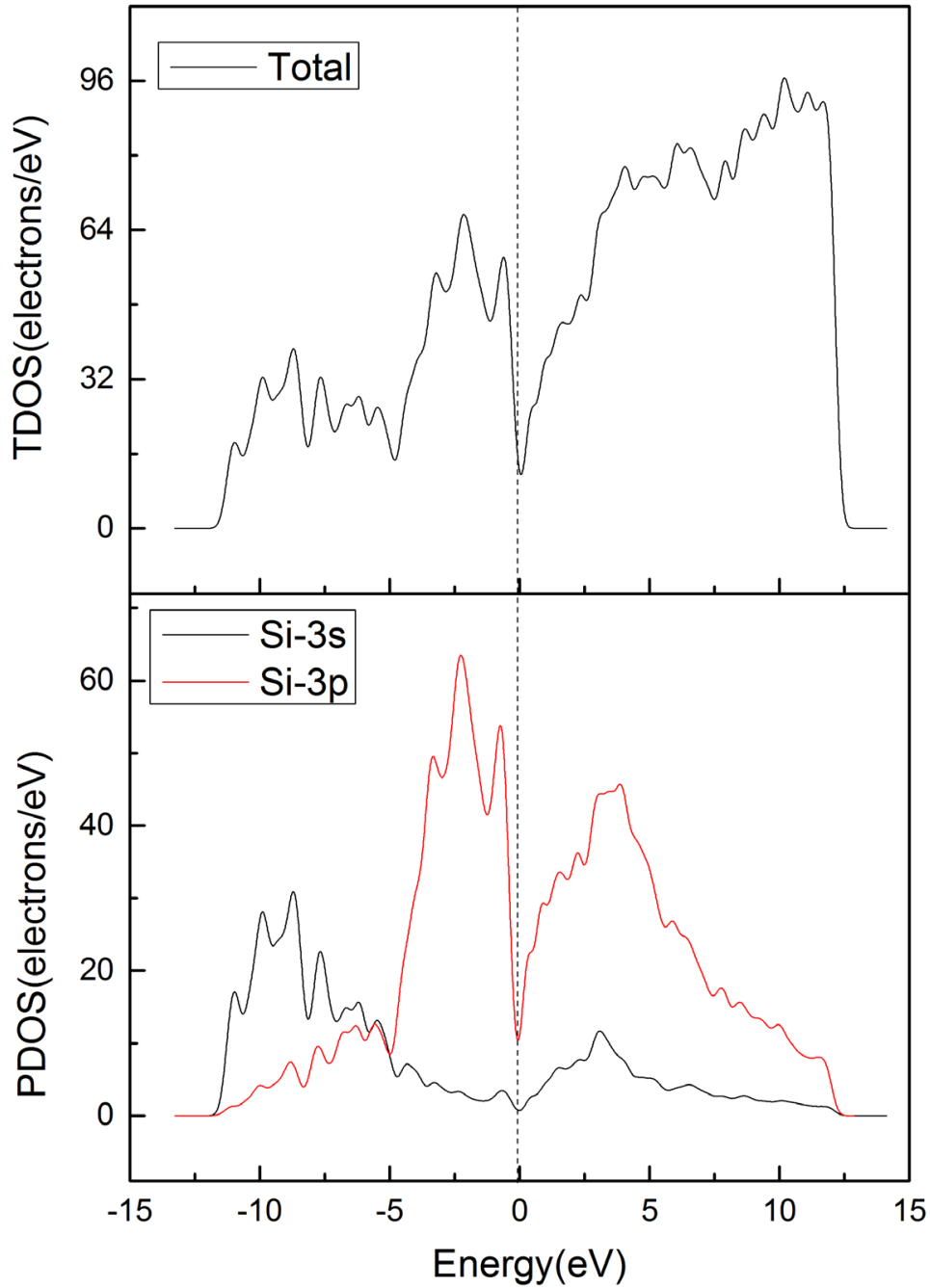

(b)

Figure 3. Band structure (a) and density of states (b) of silicon nanotube. 




(a)

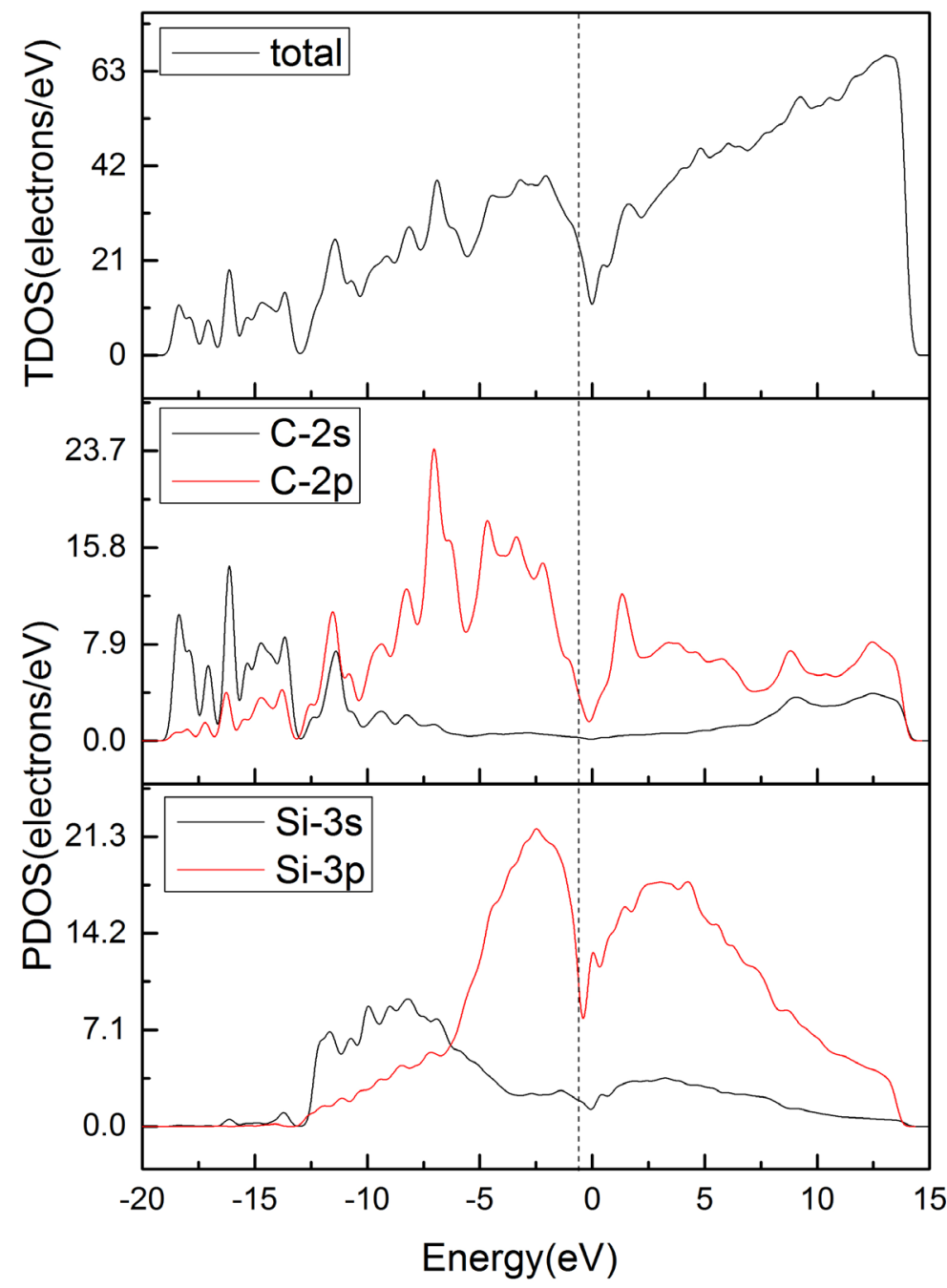

(b)

Figure 4. Band structure (a) and density of states $(b)$ of $(6,6)-(6,6) \mathrm{Si} / \mathrm{CNT}$. 


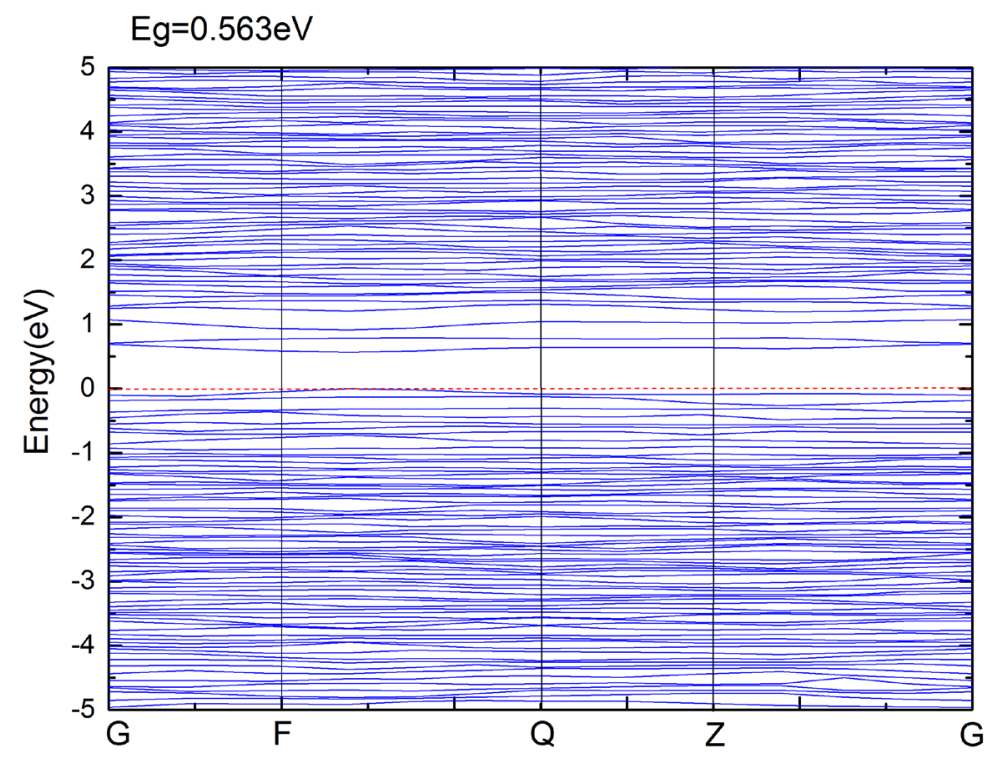

(a)

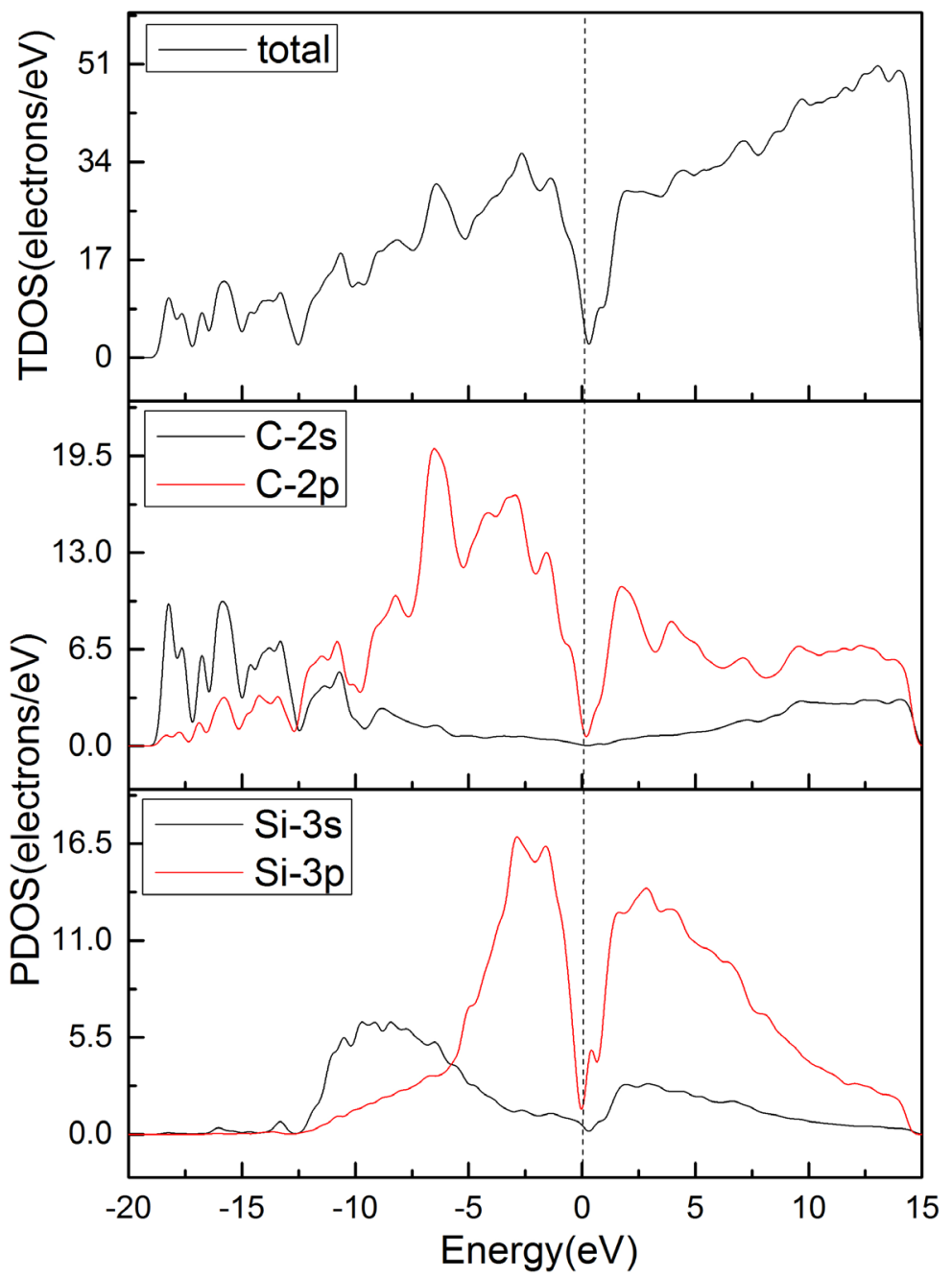

(b)

Figure 5. Band structure (a) and density of states (b) of $(4,4)-(6,6) \mathrm{Si} / \mathrm{CNT}$. 
the bonding is mainly formed by sp hybridization. In distant region far from Fermi level, the sp hybridization of $\mathrm{s}$ electrons and $\mathrm{p}$ electrons for SiNTs relatively remains obvious. The lower valence band is mainly formed from $\mathrm{Si}-3 \mathrm{~s}$ states, in which the Si-3p states exert a weak influence. Besides, the upper valence band mainly originates from $\mathrm{Si}-3 \mathrm{p}$ states.

Figure 4 and Figure 5(a) point out that the Si/CNTs exhibit semiconductor with small band gaps, less than $1 \mathrm{eV}$. The $(6,6)-(6,6) \mathrm{Si} / \mathrm{CNT}$ s have a direct band gap of $0.093 \mathrm{eV}$ (Figure $4(\mathrm{a})$ ), which is narrower than that of single-walled armchair $(6,6)$ SiNTs. The bottom of the conduction band and the top of valence band of the nanotube are located between the point $\mathrm{F}$ and $\mathrm{Q}$ of its Brillouin zone. With the top of valence band and the bottom of the conduction band between the point $\mathrm{F}$ and $\mathrm{Q}$, the $(4,4)-(6,6) \mathrm{Si} / \mathrm{CNT}$ s make a direct band gap of $0.563 \mathrm{eV}$, which is larger than that of $(6,6)$ SiNTs.

To make further study on the electronic structure of Si/CNTs, the partial density of states (PDOS) of $\mathrm{C}$ atoms and $\mathrm{Si}$ atoms in the nanotube section are investigated (in Figure 4 and Figure 5(b)). The PDOS for $(4,4)-(6,6) \mathrm{Si} / \mathrm{CNTs}$ are similar. Based on the curve, it can be seen that the bottom of the conduction band and the top of valence band are occupied by Si-3p states and C-2p states, in which the Si-3s states make a weak influence. As it shows, near the Fermi level and in the high energy region, about from $-5 \mathrm{eV}-5 \mathrm{eV}$, the quantity of split energy levels for Si/CNTs all decrease to some extent compared with SiNTs. Besides, the bonding is mainly formed by interaction of C-2p states and Si-3p electronic states. However, in the low energy region of valence band (left of the Fer$\mathrm{mi}$ level), about from $-20 \mathrm{eV}--5 \mathrm{eV}$, as certain band width exists between C-2s states and C-2p states, $\mathrm{Si}-3 \mathrm{~s}$ states and $\mathrm{Si}-3 \mathrm{p}$ states, yet is mainly from the interaction among s electrons. However, the action of $\mathrm{p}$ electrons is relatively weaker, which exhibits sp hybridization. In particular, the figure also shows that compared with $(6,6)-(6,6) \mathrm{Si} / \mathrm{CNTs}$, the hybridization strength of $(4,4)-(6,6)$ $\mathrm{Si} / \mathrm{CNTs}$ decrease slightly, and density peak strength of total electronic state is all weakened, indicating that the interelectronic locality for $(4,4)-(6,6) \mathrm{Si} / \mathrm{CNTs}$ is relatively weaker. In the vicinity of Fermi level, the peak shape of Si/CNTs is basically similar, with key distinction being caused by the changes of Si-3p electrons near Fermi level.

\subsection{Dielectric Function and Absorption}

According to the law of electron transition and Krames-Kronig dispersion relation [25], the complex dielectric and optical properties are calculated, using the well-known relation:

$$
\varepsilon(\omega)=\varepsilon_{1}(\omega)+i \varepsilon_{2}(\omega)
$$

The real part of the dielectric function follows from the relation:

$$
\varepsilon_{1}(\omega)=1+\frac{8 \pi^{2} e^{2}}{m^{2}} \cdot \sum_{V, C} \int_{B Z} \mathrm{~d}^{3} K \frac{2}{(2 \pi)} \times \frac{\left|e \cdot M_{C V}(K)\right|^{2}}{\left[E_{C}(K)-E_{V}(K)\right]}
$$


The imaginary part $\varepsilon_{2}(\omega)$, in the long wavelength limit, has been obtained directly from the electronic structure calculation:

$$
\varepsilon_{2}(\omega)=\frac{4 \pi^{2}}{m^{2} \omega^{2}} \cdot \sum_{V, C} \int_{B Z} d^{3} K \frac{2}{(2 \pi)} \times\left|e \cdot M_{C V}(K)\right|^{2} \times \delta\left[E_{C}(K)-E_{V}(K)-\hbar \omega\right]
$$

$M_{C V}(K)$ are the transition moments elements.

The absorption coefficient is given by the following relation:

$$
\alpha(\omega)=\sqrt{2} \omega\left[\sqrt{\varepsilon_{1}^{2}(\omega)+\varepsilon_{2}^{2}(\omega)}-\varepsilon_{1}(\omega)\right]^{1 / 2}
$$

$\hbar, \omega, K$ are Planck constant, the angular frequency and reciprocal vector, respectively. Subscript $\mathrm{C}$ and $\mathrm{V}$ represent the conduction band and the valence band of nanotubes, and $\mathrm{BZ}$ is the first Brillouin zone. $E_{C}(K)$ and $E_{V}(K)$ represent the intrinsic level of the conduction band and the valence band. $\left|e \cdot M_{C V}(K)\right|^{2}$ is Momentum transition matrix element of Transition electrons.

To further analyze the optical properties for the two types of Si/CNTs, Figure 6 and Figure 7 presents the real part and the imaginary part of dielectric function and absorption for single-wall armchair $(6,6)$ SiNTs, $(6,6)-(6,6) \mathrm{Si} / \mathrm{CNTs}$ and $(4,4)-(6,6) \mathrm{Si} / \mathrm{CNT}$. Figure $6(\mathrm{a})$ presents that the static dielectric constant for single-wall armchair $(6,6)$ SiNTs is 32.188 , and a dielectric absorption peak appears near $3.307 \mathrm{eV}$ with increase of energy. The static dielectric constants of $(6,6)-(6,6) \mathrm{Si} / \mathrm{CNTs}$ and $(4,4)-(6,6) \mathrm{Si} / \mathrm{CNTs}$ are 53.043 and 11.281, respectively, which also shows that a dielectric absorption peak appears when the energy of $(4,4)-(6,6) \mathrm{Si} / \mathrm{CNT}$ increases to near 1.547 . According to the inversely proportional relationship between the dielectric constant and the energy gap width of semiconductor materials, the dielectric constant of $(6,6)-(6,6)$ $\mathrm{Si} / \mathrm{CNT}$ s increase as its energy gap width decreases, while the dielectric constant of $(4,4)-(6,6) \mathrm{Si} / \mathrm{CNT}$ decrease as its energy gap width increases. This result is consistent with the calculations of energy band structure. Analysis finds that the structure of Si/CNTs can change the dielectric absorption width of real part of SiNTs.

Figure 6(b) presents the imaginary part of dielectric function of SiNTs and $\mathrm{Si} / \mathrm{CNTs}$ are presented in. Based on the figure, a sharp maximal dielectric peak in imaginary part of dielectric function for SiNTs appears in the vicinity of 0.959 $\mathrm{eV}$, which is mainly caused by intrinsic transition among Si-3p and Si-3s electrons. Besides, with the increase of energy, the dielectric peak in imaginary part appears in the vicinity of $4.359 \mathrm{eV}$ and tapers. In terms of the $(6,6)-(6,6)$ $\mathrm{Si} / \mathrm{CNTs}$, the dielectric peak strength of in low energy region increases, and conductivity is enhanced. With the increase of energy, a maximum dielectric peak appears in the vicinity of $0.443 \mathrm{eV}$ which declined. Then, a small dielectric peak appears in the vicinity of $2.243 \mathrm{eV}$. While the strength of dielectric peak for $(4,4)-(6,6) \mathrm{Si} / \mathrm{CNT}$ s is weakened to some extent, with a dielectric absorption peak appearing in the vicinity of $2.784 \mathrm{eV}$, indicating that the structure of $\mathrm{Si} / \mathrm{CNT}$ s can change the dielectric absorption width in imaginary part of SiNTs, and $(6,6)-(6,6) \mathrm{Si} / \mathrm{CNT}$ s has higher conductibility. 


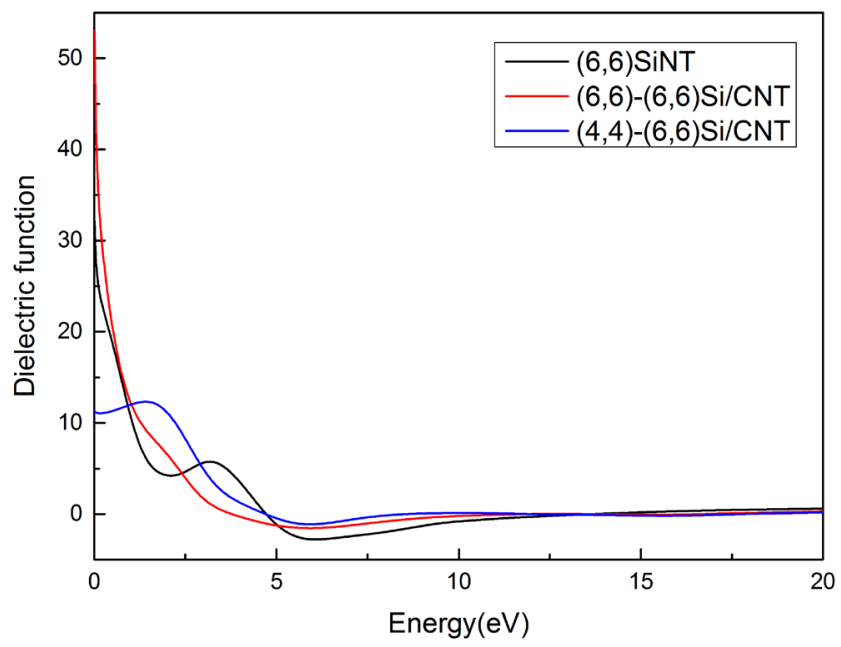

(a)

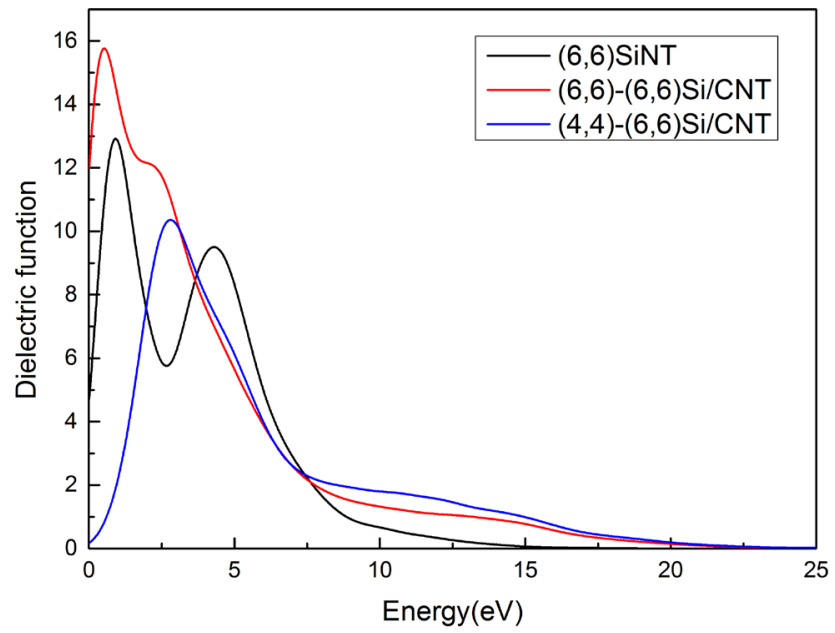

(b)

Figure 6. Dielectric function of $(6,6) \operatorname{SiNT},(6,6)-(6,6) \mathrm{Si} / \mathrm{CNT}$ and $(4,4)-(6,6)$ Si/CNT. (a) Real part; (b) imaginary part.

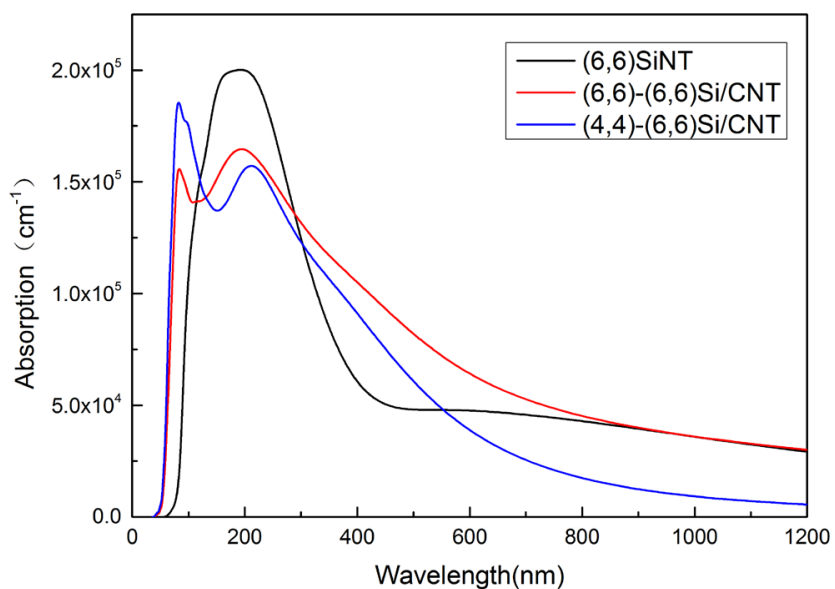

Figure 7. Absorption spectra of $(6,6) \operatorname{SiNT},(6,6)-(6,6) \mathrm{Si} / \mathrm{CNT}$ and $(4,4)-(6,6)$ $\mathrm{Si} / \mathrm{CNT}$. 
The calculate absorption coefficient of SiNTs and Si/CNTs are provided in Figure 7. Strong absorption peak appears between $100 \mathrm{~nm}-400 \mathrm{~nm}$ for absorption of $(6,6)$ SiNTs, and calculate absorption is above $1.5 \times 10^{5} \mathrm{~cm}^{-1}$, proving SiNTs have good absorption characteristics in far ultraviolet band and near ultraviolet band. In addition, the figure also shows that the absorption spectrums for $(6,6)-(6,6) \mathrm{Si} / \mathrm{CNTs}$ and $(4,4)-(6,6) \mathrm{Si} / \mathrm{CNT}$ all have strong absorption peak at $100 \mathrm{~nm}-200 \mathrm{~nm}$ and $200 \mathrm{~nm}-400 \mathrm{~nm}$, indicative of good absorption characteristics of Si/CNTs in the ultraviolet band, and the optical absorption band width of Si/CNTs is large in the high energy region near $70 \mathrm{~nm}-120 \mathrm{~nm}$, photoabsorption characteristics of far ultraviolet band is satisfactory. Besides, within $120 \mathrm{~nm}-300 \mathrm{~nm}$, the absorption coefficients of Si/CNTs all declines compared with $(6,6)$ silicon nanotube. However, absorption coefficient represents the attenuation percentage of light intensity in unit propagation length in nanotubes, so luminous efficiency of the Si/CNTs increases.

\section{Conclusion}

To conclude, in this paper, we perform first-principles calculations in the framework of density-functional theory to determine the electronic structure and optical properties of single-walled armchair $(6,6)$ SiNTs, $(6,6)-(6,6)$ $\mathrm{Si} / \mathrm{CNTs}$ and $(4,4)-(6,6) \mathrm{Si} / \mathrm{CNTs}$. The calculated results indicate that $(6,6)$ SiNTs is an indirect band gap of $0.314 \mathrm{eV}$. The $(6,6)-(6,6) \mathrm{Si} / \mathrm{CNT}$ is a direct-gap semiconductor with band gap of $0.093 \mathrm{eV}$, and $(4,4)-(6,6) \mathrm{Si} / \mathrm{CNT}$ is a direct-gap semiconductor with energy gap of $0.563 \mathrm{eV}$. The top of valence band of Si/CNTs is mainly determined by $\mathrm{Si}-3 \mathrm{p}$ and C-2p electrons, and the bottom of conduction band is occupied by the $\mathrm{C}-2 \mathrm{p}$ electrons, $\mathrm{Si}-3 \mathrm{p}$ electrons and a small amount of Si-3s electrons. It shows that the $(6,6)-(6,6) \mathrm{Si} / \mathrm{CNT}$ s has smaller band gap and higher conductibility, and $(6,6)-(6,6)$ Si/CNTs and $(4,4)-(6,6)$ $\mathrm{Si} / \mathrm{CNTs}$ have satisfactory absorption characteristics in ultraviolet band. Moreover, the results also provide instructive theoretical guidance for the applications of silicon nanotubes in optical detectors.

\section{References}

[1] Iijima, S. (1991) Helical Microtubules of Graphitic Carbon. Nature, 354, 56-58. https://doi.org/10.1038/354056a0

[2] Sammalkorpi, M., Krasheninnikov, A., Kuronen, A., Nordlund, K. and Kaski, K. (2004) Mechanical Properties of Carbon Nanotubes with Vacancies and Related Defects. Physical Review B, 70, 121-127. https://doi.org/10.1103/PhysRevB.70.245416

[3] Hou, W. and Xiao, S. (2007) Mechanical Behaviors of Carbon Nanotubes with Randomly Located Vacancy Defects. Journal of Nanoscience \& Nanotechnology, 7, 4478-4485. https://doi.org/10.1166/jnn.2007.862

[4] Kinoshita, Y., Murashima, M., Kawachi, M. and Ohno, N. (2013) First-Principles Study of Mechanical Properties of One-Dimensional Carbon Nanotube Intramolecular Junctions. Computational Materials Science, 70, 1-7. https://doi.org/10.1016/j.commatsci.2012.12.033 
[5] Chowdhury, S.C., Haque, B.Z. and Jr, J.W.G. (2014) Molecular Simulations of the Carbon Nanotubes Intramolecular Junctions under Mechanical Loading. Computational Materials Science, 82, 503-509. https://doi.org/10.1016/j.commatsci.2013.10.025

[6] Pandyan, K., Seenithurai, S. and Mahendran, M. (2012) Carbon Monoxide Adsorption on Transition Element-Doped Single Wall Carbon Nanotube. Indian Journal of Physics, 86, 677-680. https://doi.org/10.1007/s12648-012-0117-z

[7] Bistamam, M.S.A. and Azam, M.A. (2014) Tip-Growth of Aligned Carbon Nanotubes on Cobalt Supported by Alumina Using Alcohol Catalytic Chemical Vapor Deposition. Result in Physics, 4, 105-106. https://doi.org/10.1016/j.rinp.2014.07.003

[8] Li, C., Liu, Z.T., Gu, C., Xu, X. and Yang, Y. (2010) Controllable Synthesis and Growth Model of Amorphous Silicon Nanotubes with Periodically Dome-Shaped Interiors. Advanced materials, 18, 228-234.

[9] Sha, J., Niu, J.J. and Ma, X.Y. (2002) Silicon Nanotubes. Advanced Materials, 14, 1219-1221.

[10] Luo, Q., Zhang, Q., Zhang, Z., Tang, B. and Ran, Z. (2012) First Principle Study on the Structure and Electronic Property of Si Nanotubes. Micronanoelectronic Technology, 49, 152-155.

[11] Durgun, E., Tongay, S. and Ciraci, S. (2005) Silicon and III-V-Compound Nanotubes: Structure and Electric Properties. Physical Review B, 72, Article ID: 075420. https://doi.org/10.1103/PhysRevB.72.075420

[12] Lin, X., Lu, J.Z., Liu, J., Tang, Y.C. and Zhu, H.J. (2017) The Growth Model and Electronic Properties of Single- and Double-Walled Zigzag Silicon Nanotubes: Depending on the Structures. Chemical Physics, 483-484, 156-164. https://doi.org/10.1016/j.chemphys.2016.11.016

[13] Yang, Z.H., Liu, G.L., Qu, Y.D. and Li, R.D. (2015) Study on Electronic Structure and Optical Properties of Silicon Nanotube with Tensile Deformation. Journal of Synthetic Crystals, 44, 2507-2512.

[14] Yu, Z.Q., Zhang, C.H. and Lang, J.X. (2014) The Electronic Structure and Optical Properties of P-Doped Silicon Nanotube. Acta Physica Sinica, 63, Article ID: 067102.

[15] Zhang, C.H., Yu, Z.Q., Lang, J.X. and Liao, H.H. (2016) Electronic Structure and Photoelectric Properties of Al-Doped Single-Walled Armchair $(6,6)$ Silicon Nanotubes from First-Principles. Material Review, 30, 147-151.

[16] Barnard, A.S. and Russo, S.P. (2003) Structure and Energetics of Single Walled Armchair and Zigzag Silicon Nanotubes. Journal of Physical Chemistry B, 107, 7577-7581. https://doi.org/10.1021/jp0347421

[17] Fagan, S.B., Mota, R., Baierle, R.J., Paiva, G., Silva, A.J.R. and Fazzio, A. (2001) Stability Investigation and Thermal Behavior of a Hypothetical Silicon Nanotube. Journal of Molecular Structure: THEOCHEM, 539, 101-106. https://doi.org/10.1016/S0166-1280(00)00777-6

[18] Kang, Z., Li, M. and Tang, Q. (2010) Buckling Behavior of Carbon Nanotube-Based Intramolecular Junctions under Compression Molecular Dynamics Simulation and Finite Element Analysis. Computational Materials Science, 50, 253-259. https://doi.org/10.1016/j.commatsci.2010.08.011

[19] Li, M., Kang, Z., Yang, P., Meng, X. and Lu, Y. (2013) Molecular Dynamics Study on Buckling of Single-Wall Carbon Nanotube-Based Intramolecular Junctions and Influence Factors. Computational Materials Science, 67, 390-396. https://doi.org/10.1016/j.commatsci.2012.09.034 
[20] Han, D.R., Wang, L., Luo, C.L., Zhu, X.F. and Dai, Y.F. (2014) Torsional Mechanical Properties of $(n, n)-(2 n, 0)$ Carbon Nanotubes Heterojunction. Journal of Magnetic Resonance, 64, 303-313.

[21] Perdew, J.P., Burke, K. and Ernzerhof, M. (1996) Generalized Gradient Approximation Made Simple. Physical Review Letters, 77, 3865.

https://doi.org/10.1103/PhysRevLett.77.3865

[22] Monkhorst, H.J. and Pack, J.D. (1976) Special Points for Brillouin Zone Integration. Physical Review B, 13, 5188-5192. https://doi.org/10.1103/PhysRevB.13.5188

[23] Broyden, C.G. (1970) The Convergence of a Class of Double Rank Minimization Algorithms II. The New Algorithm. Journal of the Institute of Mathematics and Its Applications, 6, 222. https://doi.org/10.1093/imamat/6.3.222

[24] Yang, X.B. and Ni, J. (2005) Electronic Properties of Single-Walled Silicon Nanotubes Compared to Carbon Nanotubes. Physical Review B, 72, Article ID: 195426. https://doi.org/10.1103/PhysRevB.72.195426

[25] Shen, X.C. (1992) The Spectrum and Optical Property of Semiconductor. Science Press, Beijing, 76. 